\title{
Targeting hotspots of transmission promises to reduce malaria
}

HINXTON, UK-Over the past decade, public health officials have beefed up their efforts to eradicate malaria, rolling out insecticide-treated bed nets, increasing indoor residual spraying and boosting access to artemisinin-combination therapy. But, according to a new mathematical model, the existing interventions will not be enough to eliminate malaria in areas hardest hit by the disease (PLoS Med 7, e1000324, 2010).

In the absence of any radically new tools such as a malaria vaccine, researchers say it's time to get smart about malaria control by pinpointing the geographical areas where the disease is most likely to transmit.

"If we had the knowledge of where the hotspots were, our control tactics would be much more effective," says David Conway, who studies malarial control tactics at the UK Medical Research Council's laboratories in Gambia.

antibiotic on this bug, we're not going to have many options left," Arias says.

Historically, researchers have relied on crude genetic fingerprinting techniques and subsequent laborious fine mapping to find the genetic changes associated with drug resistance - methods that George Weinstock, associate director of the Genome Center at Washington University in St. Louis, likens to "looking for a needle in a haystack."

"Nowadays," says Weinstock, who collaborated with Arias on the sequencing, "you just sequence the whole genome and you immediately find the genome variant."

Arias's approach is not completely new. Three years ago, a team led by the Rockefeller University's Alexander Tomasz used whole-genome sequencing to track the evolution of vancomycin resistance in $S$. aureus isolates taken from a single individual (Proc. Natl. Acad. Sci. USA 104, 9451-9456, 2007).

Clearly, with costs dropping, sequencing is catching on as a powerful means of elucidating the complex pathways of drug resistance and hopefully preventing their evolution. "The technology that [Arias and others are] applying to devastating, highly drug-resistant infections is the only way of cracking a very tough nut," says Joseph Vinetz, an infectious diseases physicianscientist at the University of CaliforniaSan Diego.

Elie Dolgin
"There is a literature on targeted control in almost any other infectious disease you can think of. We just don't do it in malaria," says Philip Bejon, a clinical epidemiologist with the Kenya Medical Research InstituteWellcome Trust Collaborative Research Programme.

Starting in 1998, Bejon and his colleagues collected blood samples for up to seven years from children living in 256 homesteads in the Kilifi district of the Kenyan coast. By measuring the parasite counts in the blood, the researchers found around 20 sites of stable transmission 'hotspots', each around one kilometer in diameter and corresponding to around $15 \%$ of the population, where the incidence of malaria was on average three times as high as expected from year to year (PLoS Med. 7, e1000304, 2010).

Since first reporting the transmission hotspots in July, Bejon, in collaboration with the University of Copenhagen's Thor Theander, has tested the children's blood samples for reactivity against a polymorphic malarial surface marker called Plasmodium falciparum erythrocyte membrane protein-1. These blood tests revealed the presence of stable populations of genetically distinct malaria parasites in and around the hotspots. Looking on Google Earth or walking around the sites, Bejon couldn't see many unique characteristics associated with the hotspots. But with remote-sensing data, he found that hotspots tended to have lower levels of vegetation and a unique signature of infrared radiation.

Bejon, who reported the findings here last month at the Cold Spring Harbor LaboratoryWellcome Trust conference on Infectious Disease Genomics and Global Health, now advocates targeting these regions of elevated disease activity to wipe out the source of the parasite and, thus, provide widespread protection to the entire local communities.

\section{Getting hot}

Independent validation that blood markers can predict malarial hotspots has come from the Tanga region of northeastern Tanzania. A team led by Teun Bousema, an epidemiologist at the London School of Hygiene and Tropical Medicine, tracked children in 500 households for two years. The researchers measured malaria incidence and rates of exposure to infected mosquitoes and tested the children's blood for antibodies against the $P$. falciparum parasite. Reporting in June, they described five hotspots of active disease

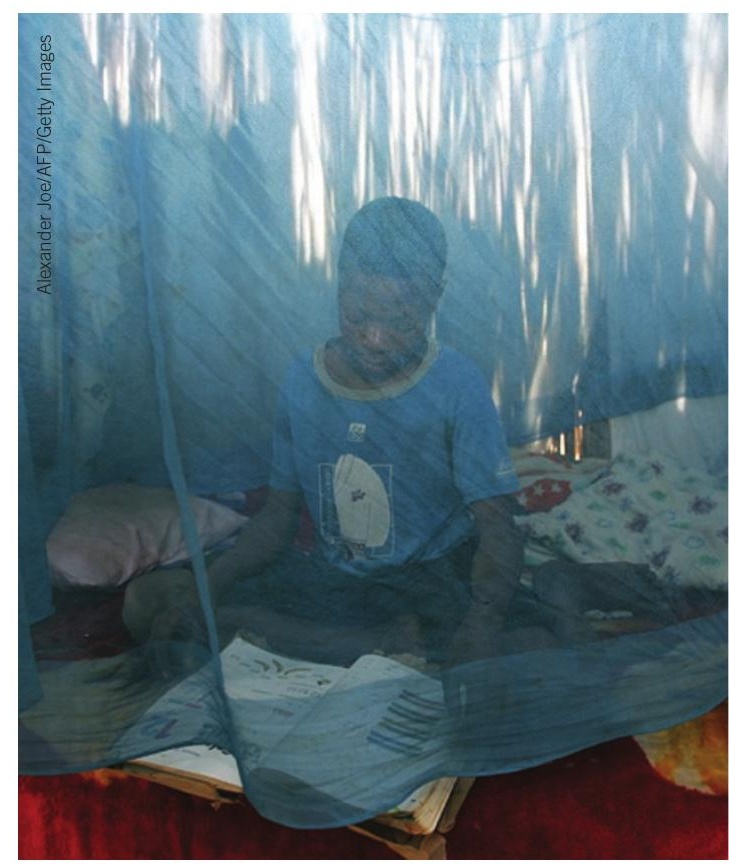

Net non-neutrality: Focused treatments.

transmission (J. Infect. Dis. 201, 1764-1774, 2010).

Since then, backed by a $\$ 100,000$ Grand Challenges Explorations grant from the Bill \& Melinda Gates Foundation, Bousema has repeated the analysis in smaller villages in Mali and found similar results.

The use of serological markersand, eventually, Bejon hopes, genomic information-should reveal patterns of local parasite transmission that are more accurate than assumptions based on the human incidences of disease. This, in turn, will allow researchers to design targeted interventions, he says.

$\mathrm{He}$ is not alone in his thinking. An unpublished mathematical model developed by Bousema in collaboration with Imperial College London's Azra Ghani and Jamie Griffin suggests that targeting the majority of the hotspots may eliminate the disease from these endemic regions.

Bousema has now applied for funding to carry out a clinical trial in which bed nets and treatments are prioritized first for people living in hotspots. "You can make a giant leap forward by putting a little bit of extra effort toward the people who are most exposed to malaria," says Bousema. "You can protect a large part of the community by eliminating the engine of transmission."

Elie Dolgin 